\title{
Editorial
}

\section{Inductively Coupled Plasma Mass Spectrometry (ICP-MS) for Environmental Monitoring and Fingerprinting}

This Hot Topic issue of "The Open Chemical and Biomedical Methods Journal" is devoted to applications of Inductively Coupled Plasma Mass Spectrometry (ICP-MS) in environmental monitoring and fingerprinting. ICP-MS has become a common method for monitoring environmental quality and processes, and for fingerprinting of natural and anthropogenic pollution sources because of its multi-elemental capability, high sensitivity, low detection limits and capability to measure isotopes. The papers herein span a wide range of interesting applications, from monitoring trace elements in atmospheric particulate matter and biota to fingerprinting sources of naturally occurring radioactive material (NORM) and historical air pollution to determining the provenance of food supply to improving sample preparation and analysis schemes. As is always the case, knowledge of the fundamentals of the instrument and technique (i.e., ICP-MS) is invaluable to obtain accurate data and avoid many of the pitfalls (e.g., matrix effects, isobaric interferences, instrumental drift) that can lead to poor data quality and misleading information. Thus, another objective of this issue is to show how different researchers effectively use ICP-MS, both solution- and laser-based, for diverse environmental samples and applications.

Environmental monitoring and fingerprinting studies often involve the determination of heavy metals and other elements for a large number of samples (dozens, if not hundreds) in complex matrices (e.g., saltwater, sediment, biota). Under these circumstances, there is great benefit from analytical schemes that speed analyses, are easy to implement, and are tolerant of difficult matrices. In the paper by Wilbur and Jones, it is shown that combining recent advances in ICP-MS technology, namely improved collision cell efficiency, aerosol dilution and discrete sampling, results in greatly improved productivity. Using a single gas (helium) and kinetic energy discrimination (larger polyatomic ions undergo more collisions in the cell and thus lose more kinetic energy relative to analyte ions) reduces measurement time. Diluting with a gas decreases water (and acid) content, yielding a hotter plasma and lower oxide formation. The discrete sampling system, essentially a clever use of a 6-port valve and a high speed pump, eliminates the need for stabilization time. The authors validate their matrix-tolerant method by examining a range of high-matrix reference materials and show accurate results for 26 elements within 2 minutes per sample.

Continuing on the easy, fast and reliable method front, Celo and coworkers present an improved microwave digestion method for fine $(<2.5 \mu \mathrm{m})$ atmospheric particulate matter using small $(7-\mathrm{mL})$ Teflon vessels to minimize the amount of acid used and subsequent dilution levels, and to increase sample throughput. Importantly, the paper demonstrates that trace metal concentrations can be accurately determined in these minute $(<<1 \mathrm{mg})$ samples without the use of hydrofluoric acid, a weak but extremely hazardous acid. The procedure was validated using reference materials, analysis of replicate samples, and by comparison with Energy Dispersive X-ray Fluorescence Spectrometry. The authors apply their improved method for samples collected within the Canadian National Air Pollution Surveillance network.

Whereas Celo et al. use trace elements determined by ICP-MS to monitor ambient air in Canada and fingerprint current pollution sources (e.g., $\mathrm{Ni}$ and $\mathrm{V}$ can indicate heavy fuel oil combustion; As and Se: coal burning; $\mathrm{Zn}$ and $\mathrm{Cu}$ : non-ferrous metallurgy), Nowinski and co-workers use trace elements, sequestered naturally in desert rock varnish, to monitor historical air pollution. In Nowinski's study, digestion was avoided altogether by analyzing the rock coating directly using laser ablation ICP-MS. In general, laser ablation methods suffer from lack of matrix-matched standards and increased variability (precision that is worse compared with solution-based methods); however, often the accuracy and precision are sufficient to meet data quality objectives for certain purposes. In such cases, the speed of analysis (LA requires little or no sample preparation), increased analyte signal (the sample is not diluted in acid), and reduced opportunities for sample contamination (less sample handling) are advantageous. Here, the authors show that rock varnish serves as a passive environmental monitor for select elements, and thus holds much potential for forensic-based air pollution studies.

For biomonitoring studies, particularly for mammals, non-lethal sampling is highly desirable; however, the approach often results in small quantities of tissue, which are further divided for multiple analyses (e.g., gas-source IRMS). The work by Bryan et al. makes use of the low detection limits and multi-element capability of ICP-MS to evaluate the use of skin biopsy samples, which can be collected in a non-lethal manner, as a monitoring tool for trace elements in the bottlenose dolphin, a sentinel for contaminants and ecosystem health monitoring of coastal marine environs. The authors examine the trace element homogeneity on a macro-scale, assessing concentrations across the animal's entire body. Whereas some elements (e.g., As and Hg) were relatively homogeneous within the organ, others (e.g., Mo, $\mathrm{Rb}, \mathrm{V}, \mathrm{Zn}$ ) were not, suggesting a standardized skin collection region would assist with comparing trace element data in the porpoise, and possibly with other cetacean studies.

A lesser known but growing application area for ICP-MS is using elements to determine the provenance of food stuffs; food fraud is highly lucrative and has become a global problem with significant economic and sometimes health impacts. Elements are incorporated into plants in varying amounts depending (primarily) on the substrate in which the plant is grown. In the case of animals, the feedstock and water sources can influence, to some extent, the elemental pattern in the animal's tissues. The paper by Watling et al. is a good primer on the subject because ICP-MS (both solution and laser ablation based) was used to 
determine the provenance of a variety of food and drink, including pork, wine, tea, coffee and olive oil. The authors validate the technology using both blind, independent trials, and actual test cases. Importantly, laser ablation, traditionally used as a method for sampling solids, has been shown to be suitable for obtaining quantitative data (elemental signatures) directly from a oilbased liquid (olive oil), and is likely applicable for similar foodstuffs (e.g., butter, cheese, chocolate) which are problematic to dissolve using conventional oxidative acid digests.

Of course, the fingerprinting of pollutants can also be based on traditional methods employing isotopes, and a contribution by Ketterer et al. is a perfect example of why ICP-MS is commonly used in such studies. ICP-MS is capable of distinguishing between isotopes of the same element at low concentrations, and at varying levels of precision, depending on, among other things, the instrument configuration, e.g., whether a multiple detector system is used. Thus, ICP-MS is well-suited for isotope dilution and isotope fingerprinting studies. Ketterer and coworkers use both: isotope dilution using ${ }^{242} \mathrm{Pu}$ for quantitation purposes and isotope fingerprinting using ${ }^{240} \mathrm{Pu} /{ }^{239} \mathrm{Pu}$ atom ratios for sourcing purposes. Their isotopic data, derived from a sector field ICP-MS revealed that Chernobyl-derived $\mathrm{Pu}$ is present in the Black Sea's Danube Delta sediments, evidently resulting from deposition into the Danube catchment basin followed by erosion and fluvial transport.

A good way to conclude this issue is with an isotopic tracing case study, which is the focus of another contribution by Ketterer and coworkers. Lead $(\mathrm{Pb})$ is a prime candidate for ICP-MS studies because it has multiple sources in the environment: crustal or "background" $\mathrm{Pb}$, so-called "common" $\mathrm{Pb}$ from industrial pollutant sources, and highly radiogenic Pb from U- and Th-rich minerals; and each source (often) has a distinct isotopic signature. Here, $\mathrm{Pb}$ isotopic compositions of contaminated sediments from the Ashtabula River (a tributary of Lake Erie located in Ohio, USA) were investigated using quadrupole ICP-MS. It was found that radiogenic $\mathrm{Pb}$ associated with anthropogenic input of NORM significantly influenced the observed $\mathrm{Pb}$ isotopic composition. The authors conclude that under some circumstances the $\mathrm{Pb}$ stable isotope method may be useful for investigating the sources, fate and transport of NORM wastes in other environmental settings, which is increasingly of concern in scientific and regulatory circles.

I hope that you find this hot-topic issue useful. I intend to use it in my instrumental analysis class to highlight the diversity of applications in which ICP-MS is being employed for environmental monitoring and fingerprinting purposes. Indeed, it demonstrates, in my opinion, that environmental monitoring and fingerprinting studies yielding meaningful and informative data and interesting conclusions are often limited by our imagination (and sometimes funding), but not by ICP-MS. Finally, I wish to express my gratitude to the authors for their excellent and valuable contributions to this hot topic issue of "The Open Chemical and Biomedical Methods Journal" and to the ICP-MS research field in general.

James Cizdziel (Guest Editor) Department of Chemistry and Biochemistry University of Mississippi P.O. Box 1848 University, MS 38677

USA

E-mail: cizdziel@olemiss.edu 